\title{
488.
}

\section{NOTE ON A RELATION BETWEEN TWO CIRCLES.}

[From the Quarterly Journal of Pure and Applied Mathematics, vol. XI. (1871), pp. 82, 83.]

Consider any two circles $O, Q$; and let $A C, B D, A^{\prime} D^{\prime}, B^{\prime} C^{\prime}$ be the common tangents touching the circles in the points $A, A^{\prime}, B, B^{\prime}, C, C^{\prime}, D, D^{\prime}$ : the locus of a point $P$ such that the pairs of tangents from it to the two circles respectively form a harmonic pencil, is a conic through the 8 points $A, A^{\prime}, B, B^{\prime}, C, C^{\prime}, D, D^{\prime}$; but this conic may break up into two lines, viz. if (as in the figure) the points $A, B^{\prime}, D^{\prime}, D$ are in a line, then the points $C, C^{\prime}, A^{\prime}, B$ will be in a symmetrically situated line, and the conic breaks up into this pair of lines, meeting suppose in $K$. The condition

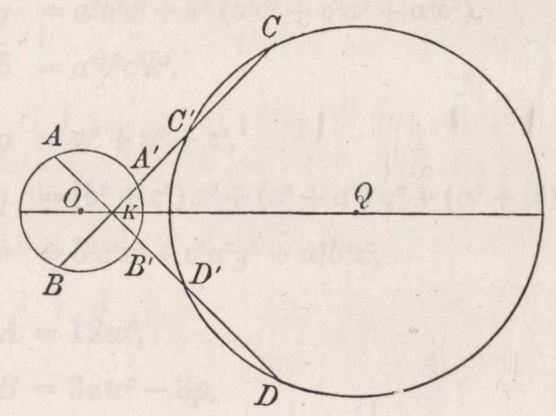

for this, if $a, a^{\prime}$ are the distances of the centres from a fixed point in the line of centres, and if the radii are $c, c^{\prime}$, is readily found to be

$$
\left(a-a^{\prime}\right)^{2}=2\left(c^{2}+c^{\prime 2}\right)
$$

Suppose in general, that (given any two conics) the point $P^{\prime}$ is the intersection of the polars of $P$ in regard to the two given conics respectively; then if $P$ describes 
a line, the locus of $P^{\prime}$ is a conic passing through the three conjugate points of the given conics; if, however, the line which is the locus of $P$ pass through one of the conjugate points, then the conic the locus of $P^{\prime}$ breaks up into a pair of lines, one of them a fixed line through the other two conjugate points, the other of them a line through the first-mentioned conjugate pcint. That is, if the locus of $P$ be a line through a conjugate point, the locus of $P^{\prime}$ is a line through the same conjugate point; but in every other case the locus of $P^{\prime}$ is a conic.

Reverting to the figure of the two circles, in order that it may be possible that the two lines $A D$ and $B C$ may be loci of points $P, P^{\prime}$, related as above, it is necessary that $K$ shall be a conjugate point of the two circles; that is, if the two circles intersect in points $\Lambda, \Lambda^{\prime}$ lying symmetrically in the radical axis, which meets, suppose, the line of centres in $M$, then it is necessary that $K$ shall be one of the anti-points of $\Lambda, \Lambda^{\prime}$; or, what is the same thing, the distance $K M$ must be $=i$ into $M \Lambda$ or $M \Lambda^{\prime}$; this condition, if as above $\left(a-a^{\prime}\right)^{2}=2\left(c^{2}+c^{\prime 2}\right)$, implies $c^{2}=c^{\prime 2}$, and we have then $\left(a-a^{\prime}\right)^{2}=4 c^{2}$, that is, the circles must be equal, and the distance of the centres must be twice the radius, or, what is the same thing, the circles must be equal circles touching each other; when this is so, the two lines $A D, B C$ (being then lines at right angles to each other intersecting in the point of contact), have, in fact, the abovementioned relation. And it thus appears that given two circles, the necessary and sufficient conditions for the coexistence of the properties mentioned in the theorem are that they shall be equal circles touching each other. 